\title{
Concomitant use of Impella while on peripheral veno-arterial extracorporeal membrane oxygenation: de-escalate and ambulate
}

\author{
Letizia Fausta Bertoldi ${ }^{1}$, Luca Bertoglio ${ }^{2}$, Federico Pappalardo $^{3}$ \\ ${ }^{1}$ Cardiac Intensive Care Unit, Advanced Heart Failure and Mechanical Circulatory Support Program, ${ }^{2}$ Division of Vascular Surgery, "Vita - Salute" \\ University, IRCCS San Raffaele Scientific Institute, Milan, Italy \\ Correspondence to: Letizia Fausta Bertoldi, MD. San Raffaele Scientific Institute, Via Olgettina 60, 20132 Milan, Italy. Email: bertoldi.letiziafausta@hsr.it.
}

Submitted Jun 27, 2018. Accepted for publication Sep 20, 2018.

doi: $10.21037 /$ acs.2018.10.15

View this article at: http://dx.doi.org/10.21037/acs.2018.10.15

\section{Clinical vignette}

A 54-year-old man with previous history of coronary artery disease, was admitted to our Cardiac Intensive Care Unit for out-of-hospital refractory cardiac arrest. At arrival, the patient was intubated and under mechanical external chest compression, and was promptly supported with veno-arterial extracorporeal membrane oxygenation (V-A ECMO) and intra-aortic balloon counterpulsation (IABP). The next day, the patient was awake and neurological examination was unremarkable; coronary angiography showed left main ulcerated plaque, treated with implantation of two drug eluting stents. During the procedure, IABP was upgraded to femoral Impella 2.5 to maximize left ventricle unloading. After 10 days, the attempt to wean from V-A ECMO failed due to acute pulmonary edema, therefore femoral Impella 2.5 was upgraded to Impella 5.0 via axillary surgical access. With Impella providing $4.4 \mathrm{~L} / \mathrm{min}$, it was possible to remove V-A ECMO the day after. During the following month, the patient was progressively weaned from mechanical ventilation and mobilized, but failed in showing myocardial recovery with persistent severe left ventricular dysfunction and was then referred for Heart Transplantation.

\section{Surgical techniques}

\section{Preparation}

The Impella 5.0 device was designed for a trans-axillary insertion and consists of a $21 \mathrm{~F}$ pump motor and a $9 \mathrm{~F}$ catheter diameter. The device can be deployed by means of both the left and right axillary arteries; the left axillary artery is preferable because it provides better angulation for catheterization of the left ventricle, and unobstructed left carotid artery flow. The patient is positioned supine, with the ipsilateral arm abducted.

\section{Exposition}

Under general anesthesia, a small transverse infraclavicular incision is performed. The fibers of the pectoralis major muscle and clavipectoral fascia are incised, exposing the pectoralis minor muscle, which is divided or laterally retracted. Arterial branches of the thoraco-acromial trunk are divided to gain full access to the axillary vessels: the axillary artery lies deep to the vein and is isolated. Heparin is administered with a goal of maintaining an activated clotting time $>250$ seconds.

\section{Operation}

A longitudinal incision is made and an end-to-side anastomosis with a $10 \mathrm{~mm}$ Dacron graft is fashioned. Gluing of the anastomosis is advisable. The conduit is clamped proximally. A small counter incision (1 cm long) is performed on the deltopectoral groove at 5-7 cm laterally to the axillary access. The conduit is then trimmed to the appropriate length and tunneled. A tourniquet is placed at the proximal level of the conduit in order to provide hemostasis during catheter insertion. The $23 \mathrm{~F}$ peel-away introducer sheath is introduced on the distal edge of the conduit. Two graft locks are placed onto the conduit and introducer sheath to provide further hemostasis.

Under fluoroscopic control, the introducer valve is 
punctured with a 0.035 Storq steerable guidewire (Cordis Corp, CA, USA) that is advanced in the aorta. In order to cross the aortic valve, an Amplatz Left 1.0 5F (Cordis Corp, CA, USA) catheter is employed and placed in the left ventricle. A right anterior oblique view is usually used in order to ascertain the graft portion of the catheter. The guidewire is exchanged with a 0.018 " V18 ControlWire guidewire (Boston Scientific, MA, USA) and the Amplatz catheter is removed.

The Impella catheter, a mono-rail system, is then introduced over the guidewire and the device is positioned in the left ventricle across the aortic valve. Correct positioning is controlled through both fluoroscopy and trans-esophageal echocardiography simultaneously. The guidewire is withdrawn and flow pressure is checked through the Impella monitor.

\section{Completion}

To remove the peel away introducer sheath, the graft is clamped with the tourniquet, the two graft locks are first released and then the peel away introducer is removed. The conduit is cut circumferentially to a sufficient length to allow placement of two silicone occlusion plugs (about $5 \mathrm{~cm}$ ) that are inserted between the catheter shaft and the conduit and fixed in place with multiple Ti-Cron threads in order to control the back bleeding. The remaining distal portion of the graft is advanced onto the Impella catheter shaft and outside of the skin incision, and is finally cut lengthwise in order to be completely removed. The Impella is secured on the skin by means of the repositioning unit suture pads and the surgical incision is readily closed.

\section{Comments}

\section{Clinical results}

Short-term mechanical circulatory support utilization has increased rapidly over the last years and it is associated with a decrease in mortality in patients with cardiogenic shock (1).

Particularly, minimally invasive left ventricular assist device (LVAD) therapy using the Impella 5.0 via axillary surgical access demonstrated to be attractive option in acute decompensated heart failure because it allows for transition to recovery or to advanced surgical therapies such as permanent LVAD and heart transplantation (2).

In the setting of INTERMACS 1 patients requiring V-A ECMO support, mortality remains high with in- hospital rate of $50 \%$ to $60 \%$ and 6-month survival as low as $30 \%$ (3). This is due to critical status but also to ECMOrelated complications (left ventricular overload, bleeding, infections). Our group has shown that concomitant implantation of Impella and V-A ECMO might improve survival as compared to ECMO alone (4).

Thus, Impella 5.0 implantation while on ECMO support, allows a safe and early weaning, avoiding ECMOrelated complications.

\section{Advantages}

Significant advantages of this approach include improved left ventricular unloading (that facilitates myocardial recovery), lower anticoagulation need, and the potential for early extubation, ambulation and physiotherapy. This reduces infections and bed-related complications and allows to share decisions with the patient. Previous reports have shown that early mobilization with the device in place have led to successful weaning strategies or bridging to durable LVAD and may be associated with improved survival (5). Furthermore, Impella 5.0 support allows to evaluate right ventricular function and pulmonary vascular resistance in a bridge to decision strategy (LVAD or heart transplantation).

\section{Caveats}

The management of mechanical circulatory support is complex and requires a multidisciplinary approach. The availability of skilled intensivists, cardiologists, vascular surgeons and nurses is crucial for the management of these patients. For this reason, patients with cardiogenic shock should be centralized in a high-volume MCS center in order to lower as much as possible mortality and morbidity rate.

\section{Acknowledgements}

None.

\section{Footnote}

Conflicts of Interest: The authors have no conflicts of interest to declare.

\section{References}

1. Stretch R, Sauer CM, Yuh DD, et al. National trends 
in the utilization of short-term mechanical circulatory support: Incidence, outcomes, and cost analysis. J Am Coll Cardiol 2014;64:1407-15.

2. Bansal A, Bhama J, Patel R, et al. Using the Minimally Invasive Impella 5.0 via the Right Subclavian Artery Cutdown for Acute on Chronic Decompensated Heart Failure as a Bridge to Decision. Ochsner J 2016;16:210-6.

3. Keebler ME, Haddad E, Choi C, et al. Venoarterial Extracorporeal Membrane Oxygenation in Cardiogenic Shock. JACC Heart Fail 2018;6:503-16.

Cite this article as: Bertoldi LF, Bertoglio L, Pappalardo F. Concomitant use of Impella while on peripheral venoarterial extracorporeal membrane oxygenation: de-escalate and ambulate. Ann Cardiothorac Surg 2019;8(1):160-162. doi: 10.21037/acs.2018.10.15
4. Pappalardo F, Schulte C, Pieri M, et al. Concomitant implantation of Impella ${ }^{\circledR}$ on top of veno-arterial extracorporeal membrane oxygenation may improve survival of patients with cardiogenic shock. Eur J Heart Fail 2017;19:404-12.

5. Esposito ML, Jablonski J, Kras A, et al. Maximum level of mobility with axillary deployment of the Impella 5.0 is associated with improved survival. Int J Artif Organs 2018;41:236-9. 